Document downloaded from:

http://hdl.handle.net/10251/151102

This paper must be cited as:

Tello-Oquendo, L.; Lin, S.; Akyildiz, IF.; Pla, V. (2019). Software-Defined architecture for QoS-Aware loT deployments in 5G systems. Ad Hoc Networks. 93:1-11. https://doi.org/10.1016/j.adhoc.2019.101911

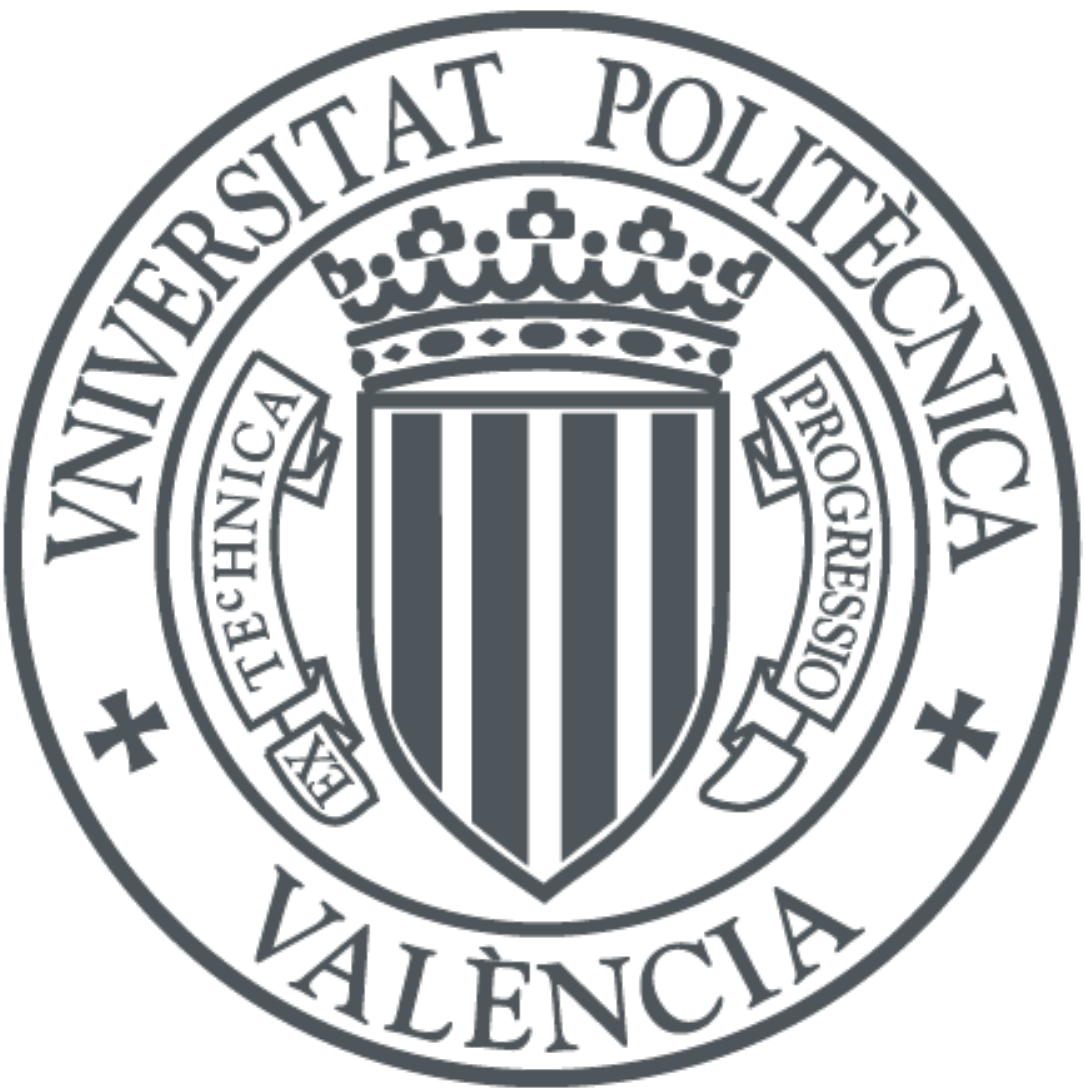

The final publication is available at

https://doi.org/10.1016/j.adhoc.2019.101911

Copyright Elsevier

Additional Information 


\title{
Software-Defined Architecture for QoS-Aware IoT Deployments in 5G Systems ${ }^{\text {is }}$
}

\author{
Luis Tello-Oquendo ${ }^{\mathrm{a}, \mathrm{b}, \mathrm{d}, *}$, Shih-Chun Lin ${ }^{\mathrm{c}}$, Ian F. Akyildiz ${ }^{\mathrm{a}}$, Vicent Pla ${ }^{\mathrm{b}}$ \\ ${ }^{a}$ Broadband Wireless Networking Laboratory, School of Electrical and Computer \\ Engineering, Georgia Institute of Technology, Atlanta, GA 30332, USA \\ ${ }^{b}$ Instituto ITACA. Universitat Politècnica de València, Valencia 46022, Spain \\ ${ }^{c}$ Intelligent Wireless Networking Laboratory, Department of Electrical and Computer \\ Engineering, North Carolina State University, Raleigh, NC 27695, USA \\ ${ }^{d}$ College of Engineering, Universidad Nacional de Chimborazo, Riobamba 060108, Ecuador
}

\begin{abstract}
Internet of Things (IoT), a ubiquitous network of interconnected objects, harvests information from the environments, interacts with the physical world, and uses the existing Internet infrastructure to provide services for information transfer and emerging applications. However, the scalability and Internet access fundamentally challenge the realization of a wide range of IoT applications. Based on recent developments of 5G system architecture, namely SoftAir, this paper introduces a new software-defined platform that enables dynamic and flexible infrastructure for $5 \mathrm{G}$ IoT communication. A corresponding sum-rate analysis is also carried out via an optimization approach for efficient data transmissions. First, the SoftAir decouples control plane and data plane for a software-defined wireless architecture and enables effective coordination among remote radio heads (RRHs), equipped with millimeter-wave (mmWave) frontend, for IoT access. Next, by introducing an innovative design of softwaredefined gateways (SD-GWs) as local IoT controllers in SoftAir, the wide diversity of IoT applications and the heterogeneity of IoT devices are easily accommodated. These SD-GWs aggregate the traffic from heterogeneous IoT devices and
\end{abstract}

\footnotetext{
This work was supported by the US National Science Foundation (NSF) under Grant No. 1547353, in part by the Ministry of Economy and Competitiveness of Spain under Grants TIN2013-47272-C2-1-R and TEC2015-71932-REDT.

* Corresponding author

Email address: luis.tello@unach.edu.ec (Luis Tello-Oquendo)
} 
perform protocol conversions between IoT networks and radio access networks. Moreover, a cross-domain optimization framework is proposed in this extended SoftAir architecture concerning both upstream and downstream communication, where the respective sum-rates are maximized and system-level constraints are guaranteed, including (i) quality-of-service requirements of IoT transmissions, (ii) total power limit of mmWave RRHs, and (iii) fronthaul network capacities. Simulation results validate the efficacy of our solutions, where the extended SoftAir solution surpasses existing IoT schemes in spectral efficiency and achieves optimal data rates for next-generation IoT communication.

Keywords: Internet of things (IoT), software-defined networking (SDN), 5G cellular systems, software-defined gateway, millimeter-wave transmissions, cross-domain optimization.

\section{Introduction}

Internet of Things (IoT) is one of the most transformative and disruptive technologies for the fifth-generation (5G) wireless systems. It has the potential to change the world radically due to its capacity to provide connectivity 5 to anyone/anything at any time and any location. It is anticipated that there will be 20 billion IoT connected devices by 2023 and the global monthly mobile data traffic will achieve 110 exabytes $\left(10^{18}\right.$ bytes $)[1$. However, facing this vast number of IoT devices is a challenging task, in particular, the ubiquitous information transmissions through the backbone networks, such as cellular systems. Moreover, the heterogeneity of IoT devices and the hardware-based, inflexible cellular infrastructure impose even more significant challenges to enable efficient IoT communication.

Current IoT solutions rely on low-power wide area (LPWA) networks, which complement traditional cellular and short-range wireless technologies in addressing IoT applications. Several technologies, such as LoRa, NB-IoT, SIGFOX, have been developed and designed solely for applications with very limited demands on throughput, reliability, or quality-of-service (QoS) [2]. However, with- 
out a central regulation among these LPWA technologies, existing IoT solutions cannot support highly diverse QoS requirements from an increasing number of IoT applications. Furthermore, due to currently fixed and hardware-based infrastructure, no existing work has considered the joint architectural design of IoT networks and radio access networks (RANs), and the provision of reliable and efficient upstream/downstream IoT transmissions.

To adequately address the above challenges in $5 \mathrm{G}$ IoT, we introduce a new architecture based on the so-called SoftAir [3] to support flexible IoT infrastructure. Also, we propose a sum-rate optimization framework upon SoftAir to yield optimal spectral efficiency in IoT communication. Specifically, inspired by wireless software-defined networking [4, 5], we first propose the SoftAirbased architecture, which decouples control and data planes for an open, programmable, and virtualizable wireless forwarding infrastructure. It allows real time network information accessibility and global optimized control. The data plane consists of software-defined RANs (SD-RANs) and software-defined core networks; the control plane has network management tools and user applications. In SD-RANs, SoftAir centralizes the communication functionality in the baseband server (BBS) pool and enables efficient coordination among hardwarebased remote radio heads (RRHs), equipped with millimeter-wave (mmWave) frontend and multiple antennas. Moreover, the cooperativeness of SoftAir facilitates the implementation and aggregation of virtual base stations (VBSs) at the BBS pool to enhance the performance of the system by joint orchestration/optimization [6].

In addition, we propose software-defined gateways (SD-GWs) as local IoT controllers in SoftAir. They can be deployed for satisfying the massive connectivity and diverse traffic generated by a vast number of IoT devices. SDGWs, serving as the bridge between IoT networks and SD-RANs, provide intensive data aggregation from heterogeneous IoT devices, manage and orchestrate IoT communication, and perform protocol conversions between IoT networks and SD-RANs. This innovative design of SD-GWs enables smooth and ubiquitous information transmissions, traversing between IoT and backbone net- 
works. Moreover, upon the SoftAir architecture, we propose a sum-rate optimization framework that maximizes upstream/downstream data rates of IoT communication thus offering low latency and efficient spectrum usage. Based on the physical-layer modeling of mmWave multi-input and multi-output (MIMO) transmissions, the objective is to maximize total data rates from/to SD-GWs through optimal associations of SD-GWs and mmWave RRHs and the respective pre-coding schemes, while guaranteeing (i) QoS requirements from diverse IoT applications, (ii) total power limit of mmWave RRHs, and (iii) fronthaul capacity constraints between the BBS pool and mmWave RRHs.

Our main contributions are summarized as follows:

- A 5G SoftAir-based architecture is introduced to provide efficient, ubiquitous IoT transmissions by supporting a unified software-defined platform for both IoT networks and cellular systems.

- An innovative design of SD-GWs as local IoT controllers is proposed to orchestrate IoT devices and perform protocol conversions between IoT networks and SD-RANs.

- Upon the SoftAir architecture, a sum-rate optimization framework is proposed that achieves efficient spectrum usage and optimal data rates for both upstream and downstream IoT communication.

Simulation results show that our solutions outperform existing IoT infrastructure (with hardware-based architectures and fixed IoT-RAN associations), and achieves optimal rates of $100 \mathrm{Mb} / \mathrm{s}$ and $430 \mathrm{Mb} / \mathrm{s}$ for upstream and downstream transmissions, respectively. Regarding densely-deployed IoT, we also examine both the impact of increasing the number of mmWave RRHs with a fixed number of antennas and the impact of increasing the number of antennas with a fixed number of mmWave RRHs on the achievable sum-rates. The rest of the paper is organized as follows. Section 2 presents the related work. Section 3 introduces the unified software-defined platform for $5 \mathrm{G}$ IoT communication and the system model used in this study. Section 4 proposes the sum-rate analysis 
for $5 \mathrm{G}$ IoT transmissions via an optimization approach. Section 5 gives the numerical results, and Section [6 concludes the paper. [

\section{Related Work}

While individually, significant research has been carried out in the domains of SDN [7, 8, 9] and IoT [10, 11, a combination of the two remains an open research area and attracts great community attention over the past few years. In 12 , SDN is used to enable IoT networks where a central controller translates handle heterogeneous IoT flows. However, the work lacks a specific design of the inner workings of the controller and evaluation of the proposed high-level architecture.

Notations: Throughout this paper, boldface lower and upper case symbols represent vectors and matrices, respectively; $\mathbf{I}_{x}$ denotes the $x \times x$ identity matrix; $\mathbb{C}^{x, y}$ denotes the set of $x \times y$ complex matrices. The trace, transpose, and Hermitian transpose operators are denoted by $\operatorname{tr}(\cdot),(\cdot)^{\mathrm{T}}$, and $(\cdot)^{\mathrm{H}}$, respectively. We use $\mathcal{C N}(\mathbf{X}, \mathbf{Y})$ to denote the circular symmetric complex Gaussian distribution with mean matrix $\mathbf{X}$ and covariance matrix $\mathbf{Y}$; the distribution function of a uniform random variable is denoted by $\mathcal{U}(\cdot)$, the distribution function of a normal random variable with mean $x$ and variance $\sigma^{2}$ is denoted by $\mathcal{N}(x, \sigma)$, and $\sim$ stands for "distributed as". $\|\mathbf{x}\|$ denotes the Euclidean norm of a complex vector $\mathbf{x} ;|z|$ denotes the magnitude of a complex number $z$. 
In [14, the authors propose a novel SDN-orchestrated network virtualization. layer, the SDN-enabled network switches with an IoT gateway (to connect them to the middle layer) are contained. Virtualization techniques are used to design the network OS with the aim of achieving an IoT-optimized network. This 


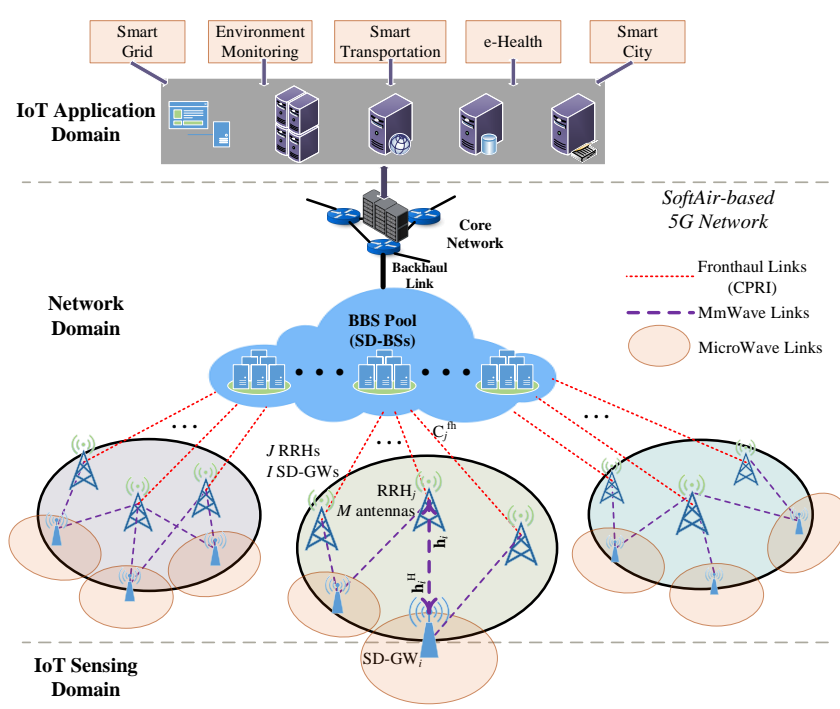

Figure 1: Unified software-defined platform for 5G IoT communication. large scale.

The sensing domain enables IoT devices to interact and communicate with each other, through the data collection technologies such as wireless sensor networks, RFID, ZigBee or near-field communication. The network domain builds 


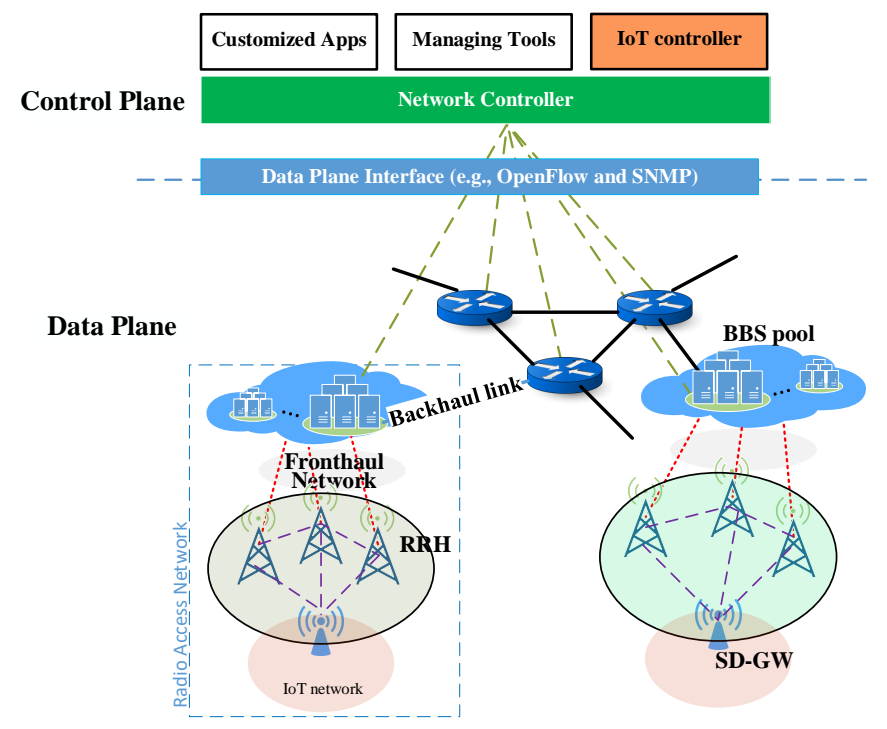

Figure 2: A SoftAir architecture for IoT communication within 5G systems.

on SoftAir; it consists of three parts as depicted in Fig. 2 (i) the centralized BBS pool, which connects to the core network via backhaul links; it has software-defined BSs (SD-BSs) from real-time virtualization technology that allow for software-implemented baseband units (e.g., digital processing tasks), (ii) mmWave RRHs plus antennas, which are remotely controlled by SD-BSs and serve SD-GWs' transmissions, and (iii) low-latency high-bandwidth fronthaul links (fiber or microwave) using the common public radio interface (CPRI) for an accurate, high-resolution synchronization among mmWave RRHs. The objective of this network domain is to transfer the data collected from the sensing domain to the remote destination. Finally, the application domain is responsible for data processing and the provision of a wide variety of applications and services.

Regarding the security and privacy sublayer, transversal to all the domains, it is set to protect the communications throughout the entire system and to ensure all trusted devices/data will be operated/processed. Concretely, an identity-based networking service is proposed that use flow rules to control 
the traffic arrivals through security devices. The IEEE 802.1X protocol can be implemented for switch-port based network access control, jointly with the lightweight directory access protocol (LDAP) for authentication along with an access control server that implements radius, AAA authentication, and network access control lists (flow rules to control traffic in and out). Furthermore, this sublayer also implements secure shell (SSH), HTTPS, and IPSEC tunneling for remote access and monitoring.

Additionally, the controller architecture deploys several virtualized components such as task-resource matching, service specification, and flow scheduling to address the heterogeneous and dynamic needs of IoT applications and services. The task-resource matching component maps the task request of IoT applications or services onto the existing resources in IoT heterogeneous networks. The service solution specification component maps the features of devices and services involved in the communication to precise requirements for devices, networks, and application constraints. The flow scheduling component schedules the flows that satisfy the specified requirements. Note that the heterogeneity of the networks and various QoS requirements of flows becomes the scheduling and coordination of the resources in IoT networks complex. Preprocessing and analysis will be performed at the edge of the networks if necessary through fog computing. Finally, we use a logically centralized management and coordination component for addressing the synchronization and inter-controller communications. By doing so, our proposed architecture will orchestrate the end-to-end communication and the necessary resources for satisfying the IoT connectivity with strict QoS requirements and energy constraints.

It is worth noting that an essential architectural component is the SD-GW, that lies between the sensing and network domain. Besides alleviating high traffic loads from tremendously heterogeneous IoT devices, these SD-GWs aggregate the data from randomly deployed IoT devices and provide Internet access to IoT networks through SD-RANs. SD-GWs also manage IoT connectivity and orchestrate IoT devices by regulating parameters in network protocols. Therefore, they can receive IoT data traffic from the IoT sensing devices and forward 
the traffic to the SoftAir SD-RAN. Depending on the communication direction, each SD-GW will either perform protocol conversions in such a way it can forward the data to the SoftAir system with the maximum achievable rate or forward the data to the IoT devices meeting the application QoS requirements. In [17, we considered the joint architectural design of IoT networks and software-defined radio access networks (SD-RANs) to the provision of reliable and efficient upstream/downstream IoT transmissions.

\subsection{System Model}

The system model of SoftAir consists of a set $\mathcal{I}=\{1, \ldots, I\}$ of SD-GWs that are served by a set $\mathcal{J}=\{1, \ldots, J\}$ of associated RRHs. All RRHs are connected to the BBS pool $\mathcal{B}$ via fronthaul links, where the $j$ th fronthaul link between the $j \in \mathcal{J}$ RRH and the $\mathcal{B}$ BBS pool has a predetermined capacity $C_{j}^{(\mathrm{fh})}$. The BBS pool performs most baseband processing tasks while transmission functions are realized by the RRHs using the processed baseband signals received from the BBS through the fronthaul transport network. The associations between the SD-GWs and RRHs can be determined based on the distance or channel gain from each RRH to each SD-GW. These RRHs are equipped with an array of $M$ antennas and communicate with the single-antenna SDGWs through mmWave links. Note that by using low-latency high-bandwidth fronthaul links, the software-defined architecture implements an accurate, highresolution synchronization and enables flexible coordination among RRHs. One $\mathrm{RRH}$ can serve a group of SD-GWs: when the $j$ th RRH is assigned to serve the $i$ th SD-GW, this RRH receives the SD-GW's processed baseband signal from the BBS pool and then modifies the pre-coding vectors accordingly.

\section{Sum-Rate Analysis for 5G IoT Communication via an Optimiza- tion Approach}

In the following, we first model the peculiarities of mmWave transmissions in the SoftAir architecture; then, we propose a protocol for mmWave $\mathrm{RRH}$ 

tween the $i$ th SD-GW and $j$ th RRH. Particularly, we detail the path-loss $l_{i}$, channel vector $\mathbf{h}_{i}$, and beamforming gain $G_{i}^{(\mathrm{BF})}$ to derive the achievable upstream rate $R_{i}^{(\mathrm{ul})}$ and downstream rate $R_{i}^{(\mathrm{dl})}$, respectively.

\subsubsection{Path-Loss}

Considering the special characteristics of mmWave propagation (such as short-range communication, inevitable blockage effects, and sparse-scattering radio patterns), the path-loss for a mmWave communication link $l_{i}$ can be modeled with three link-states: outage $\left(l_{i O}\right), \operatorname{LoS}\left(l_{i L}\right)$ or NLoS $\left(l_{i N}\right)$ [18. These three states are formulated as follows

$$
l_{i O}=0 ; \quad l_{i L}=\left(\alpha_{L} d_{i}\right)^{-\beta_{L}} ; l_{i N}=\left(\alpha_{N} d_{i}\right)^{-\beta_{N}},
$$

where $\alpha_{L}\left(\alpha_{N}\right)$ can be interpreted as the path-loss of the LoS (NLoS) link at $1[\mathrm{~m}]$ distance, and $\beta_{L}\left(\beta_{N}\right)$ denotes the path-loss exponent of the LoS (NLoS) link; from experimental results [18, $\beta_{N}$ value (can be up to 4 ) is normally higher than $\beta_{L}$ value (i.e., 2). Then, each link-state is formulated by the probabilities $\mathbb{P}_{O}, \mathbb{P}_{L}$, and $\mathbb{P}_{N}$, respectively, as

$$
\begin{aligned}
& \mathbb{P}_{O}=\max \left(0,1-\gamma_{O} e^{-\delta_{O} d_{i}}\right) \\
& \mathbb{P}_{L}=\left(1-\mathbb{P}_{O}\right) \gamma_{L} e^{-\delta_{L} d_{i}} \\
& \mathbb{P}_{N}=\left(1-\mathbb{P}_{O}\right)\left(1-\gamma_{L} e^{-\delta_{L} d_{i}}\right),
\end{aligned}
$$

where $d_{i}$ denotes the transmitter-receiver distance; the parameters $\gamma_{L}\left(\gamma_{O}\right)$ and $\delta_{L}\left(\delta_{O}\right)$ depend on both the propagation scenario and the considered carrier frequency 19 . 
Table 1: Three-state Link Path Loss Model Computation Parameters and the Occurrence Probabilities of LOS, NLOS, and Outage States at $73[\mathrm{GHz}]$ from Experimental Data in [18, 19]

\begin{tabular}{ll}
\hline Path loss model (three-state link) & $\alpha_{L}=10^{\frac{69.8}{20}}, \beta_{L}=2$ \\
Eqs. (1)-(3) & $\alpha_{N}=10^{\frac{82.7}{26.9}}, \beta_{N}=2.69$ \\
\hline & $\alpha_{L}=10^{\frac{69.8+\zeta_{L}}{20}}, \beta_{L}=2$ \\
Path loss model (three-state link) and & $\zeta_{L}[d B] \sim \mathcal{N}\left(0,5.8^{2}\right)$ \\
log-normal shadowing Eqs. (1)-(3) & $\alpha_{N}=10^{\frac{82.7+\zeta_{N}}{26.9}}, \beta_{N}=2.69$ \\
& $\zeta_{N}[d B] \sim \mathcal{N}\left(0,7.7^{2}\right)$ \\
\hline LOS-NLOS-outage probability & $\gamma_{L}=1, \delta_{L}=1 / 67.1$ \\
parameters Eq. (2) & $\gamma_{O}=\exp (5.2), \delta_{O}=1 / 30$ \\
\hline
\end{tabular}

Thus, the corresponding path-loss component of the channel is modeled as

$$
\begin{aligned}
l_{i}= & \mathbb{I}\left[U<\mathbb{P}_{L}\left(d_{i}\right)\right] l_{i L}+ \\
& \mathbb{I}\left[\mathbb{P}_{L}\left(d_{i}\right) \leq U<\left(\mathbb{P}_{L}\left(d_{i}\right)+\mathbb{P}_{N}\left(d_{i}\right)\right)\right] l_{i N}+ \\
& \mathbb{I}\left[\left(\mathbb{P}_{L}\left(d_{i}\right)+\mathbb{P}_{N}\left(d_{i}\right)\right) \leq U \leq 1\right] l_{i O},
\end{aligned}
$$

where $\mathbb{I}[x]$ is the indicator function, it returns 1 when $x$ is true, and 0 otherwise; $U \sim \mathcal{U}[0,1]$ is a uniform random variable. For computing the path-loss model, we use the parameter values at $73 \mathrm{GHz}$ as shown in Table 1 .

\subsubsection{Channel Vector}

Besides the peculiarities of mmWave transmissions [18, 20, the blockage information is not entirely feasible; therefore, we exploit the stochastic geometry analysis for modeling the mmWave channel vector [20. Specifically, we model the channel vector as $\mathbf{h}_{i}=\sqrt{l_{i} \boldsymbol{\beta}_{i}} \boldsymbol{\xi}_{i} \in \mathbb{C}^{M, 1}$, where $l_{i}$ is the large-scale pathloss in power of the mmWave communication link $i$ (which might also include log-normal shadowing), $\boldsymbol{\beta}_{i} \in \mathbb{C}^{M, M}$ is the co-variance matrix for antenna correlations in small-scale fading, and $\boldsymbol{\xi}_{i} \in \mathbb{C}^{M, 1}$ is a Gaussian vector with the zero-mean circularly symmetric Gaussian noise distribution $\mathcal{C N}\left(0, \mathbf{I}_{M}\right)$ for the fast-fading. 


\subsubsection{Beamforming}

To ensure an acceptable range of the communication in the multi-antenna mmWave transmissions, we introduce the precode vectors, i.e., beamforming weights at the RRHs, where the weight vector $\mathbf{w}_{i} \in \mathbb{C}^{M, 1}$ is the linear downlink beamforming vector at the $j$ th $\mathrm{RRH}$ corresponding to the $i$ th SD-GW. The beamforming gain is given as $G_{i}^{(\mathrm{BF})}=\mathbf{w}_{i}^{\mathrm{H}} \boldsymbol{\beta}_{i} \mathbf{w}_{i}$, with $\boldsymbol{\beta}_{i}$ being the covariance matrix of the channel response vector $\mathbf{h}_{i}$. In the case where the fading is fully correlated between the antennas, the matched filtering pre-coding method is exploited as $\boldsymbol{\beta}_{i}=\mathbf{h}_{i}^{\mathrm{H}} \mathbf{h}_{i}$ and $\mathbf{w}_{i}=\mathbf{h}_{i} /\left\|\mathbf{h}_{i}\right\|$; therefore, $G_{i}^{(\mathrm{BF})}=\left\|\mathbf{h}_{i}\right\|^{2}$.

\subsection{Protocol for MmWave RRH Discovery}

We consider a time-division duplex (TDD) mode to exploit channel reciprocity in uplink and downlink transmissions; it offers availability of timely and accurate feedback of channel state information (CSI). Also, in SoftAir the VBSs in the central BBU pool can easily share CSI associated with different users in the system.

Specifically, the time-frequency wireless resources are divided into frames, where a frame consists of $T_{f}$ seconds and $W \mathrm{~Hz}$ and leaves room of $S=T_{f} W$ transmission symbols, as shown in Fig. 3. In each frame, $D \geq 1$ out of the $S$ symbols are dedicated for allocating the RRH's reference signal (RS); the remaining $S-D$ symbols are used for payload data where $1-\kappa$ and $\kappa$ denote the fixed fractions for uplink and downlink transmissions, respectively.

Each RRH broadcasts its RS at the beginning of every frame. The RS is of duration $T_{r s} \ll T_{f}$. In each frame $i$, a mmWave $\mathrm{RRH}$ transmits the RS using beamformer $\mathbf{w}_{i}$. Each RRH needs $I$ consecutive frames to complete one cycle of spatial scanning using $I$ beamformers [21. At the receiving end, each SD-GW collect signal from $\digamma$ frames and performs non-coherent detection to detect the presence of RSs and their timings. The parameter $\digamma$ can be set to $\digamma=K I$, where $K \geq 1$ is an integer. By doing so, we ensure that, in presence of spatial scanning, each SD-GW has been covered by at least one beam and can accumulate sufficient energy for detection purposes. 


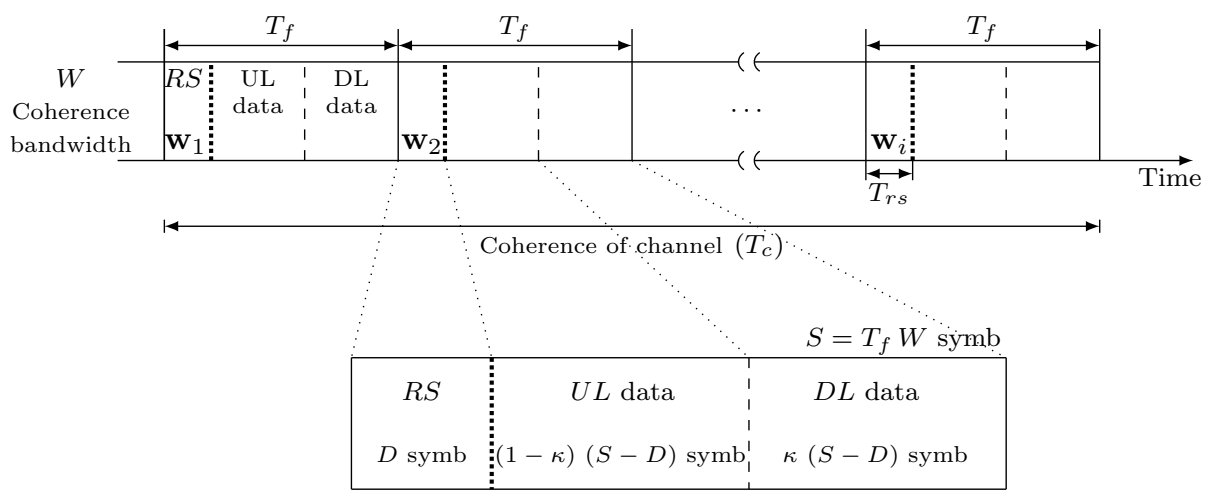

Figure 3: Frame structure with beamforming transmission of RS in TDD mode.

\subsubsection{Association Scheme}

Let $\mathcal{J}=\{1, \ldots, J\}$ and $\mathcal{I}=\{1, \ldots, I\}$ denote the set of RRHs and SDGWs in the SoftAir system, respectively. Suppose that each SD-GW is served by a specific group (cluster) of associated RRHs, and a RRH can serve multiple SD-GWs at the same time. To express the association status between RRHs and SD-GWs, we introduce the following binary variables as the indicators. Concretely, RRHs can be active to serve SD-GWs or shutdown to save the energy consumption, let $\left\{q_{j}, j \in \mathcal{J}\right\}$ denotes the activity of RRHs as

$$
q_{j}=\left\{\begin{array}{lc}
1, & \text { the } j \text { th } \mathrm{RRH} \text { is in active mode; } \\
0, & \text { otherwise }
\end{array}\right.
$$

let $\left\{g_{i j}, i \in \mathcal{I}, j \in \mathcal{J}\right\}$ denotes the association between RRHs and SD-GW as

$$
g_{i j}=\left\{\begin{array}{lc}
1, & \text { the } i \text { th } \mathrm{SD}-\mathrm{GW} \text { is served by the } j \text { th RRH; } \\
0, & \text { otherwise; }
\end{array}\right.
$$

furthermore, to characterize the group (cluster) of serving RRHs, let $\left\{N_{i j}, i \in\right.$ $\mathcal{I}, j \in \mathcal{J}\}$ be the clustering indicator as

$$
N_{i j}=\mathbb{I}\left[j \in \mathcal{N}_{i}\right],
$$


where $\mathcal{N}_{i}$ denotes the set of near RRHs for the $i$ th SD-GW which can be determined based on the distance or channel gain from RRHs to each SD-GW.

\subsection{Achievable Sum-Rate Analysis}

In the following, we first investigate channel estimation; then, we study the ergodic achievable spectral efficiency and capacity in both, the upstream and downstream IoT communication.

\subsubsection{Minimum mean-square error (MMSE) Channel Estimation}

During a training phase in TDD networks, the SD-GWs served by a cluster $\mathcal{N}_{i}$ of RRHs transmit mutually orthogonal pilot sequences which allow the BBS pool to compute the estimate $\hat{\mathbf{H}}_{i}$ of the local channel $\mathbf{H}_{i}$. While the same set of orthogonal pilot sequences might be reused among RRH clusters, the channel estimate is corrupted by pilot contamination from adjacent clusters 22. After correlating the received training signals $\breve{\mathbf{Y}}_{i}$ with the pilot sequences $\mathbf{w}_{i}$, the BBS pool acquires the noisy observation as

$$
\breve{\mathbf{Y}}_{i}^{*}=\breve{\mathbf{Y}}_{i} \mathbf{w}_{i}^{\mathrm{H}} \triangleq\left[\breve{\mathbf{y}_{1}^{*}} \cdots \breve{\mathbf{y}}_{I}^{*}\right] \in \mathbb{C}^{M\left|\mathcal{N}_{i}\right|, I},
$$

and accordingly estimates the channel vector $\mathbf{h}_{i}$. Specifically, we adopt the MMSE estimator 23] which gives the estimate $\hat{\mathbf{h}}_{i}$ of $\mathbf{h}_{i}$ as

$$
\begin{aligned}
\hat{\mathbf{h}}_{i} & =\mathbf{A}_{i} \mathbf{Q}_{i} \breve{\mathbf{y}}_{i}^{*} \\
& =\mathbf{A}_{i} \mathbf{Q}_{i}\left(\mathbf{h}_{i}+\sum_{k \in \mathcal{N}} \mathbf{h}_{k}+\frac{1}{\sqrt{\rho_{t r}}} \breve{\mathbf{n}}_{i}\right) \sim \mathcal{C N}\left(\mathbf{0}, \Phi_{i}\right),
\end{aligned}
$$

where

$$
\begin{aligned}
& \mathbf{A}_{i} \triangleq \mathbb{E}\left[\mathbf{h}_{i} \mathbf{h}_{i}^{\mathrm{H}}\right] \in \mathbb{C}^{M\left|\mathcal{N}_{i}\right|, M\left|\mathcal{N}_{i}\right|} ; \\
& \mathbf{Q}_{i}=\left(\frac{1}{\rho_{t r}} \mathbf{I}_{M\left|\mathcal{N}_{i}\right|}+\sum_{k \in \mathcal{J}} \mathbf{A}_{k}\right)^{-1} ;
\end{aligned}
$$

$\sqrt{\rho_{t r}}$ denotes the effective training signal-to-noise (SNR) ratio, $\rho_{t r}>0$;

$\breve{\mathbf{n}}_{i} \sim \mathcal{C N}\left(\mathbf{0}, \mathbf{I}_{M\left|\mathcal{N}_{i}\right|}\right) ;$

$\mathcal{N}$ denotes the set of other clusters that use the same pilot as the one adopted in cluster $\mathcal{N}_{i}$ for the SD-GW $i$;

$\mathbf{\Phi}_{i} \quad=\mathbf{A}_{i} \mathbf{Q}_{i} \mathbf{A}_{k}, k \in \mathcal{J}$. 
Applying the orthogonality of the MMSE estimate, the channel vector can be further decomposed as $\mathbf{h}_{i}=\hat{\mathbf{h}}_{i}+\tilde{\mathbf{h}}_{i}$, where $\tilde{\mathbf{h}}_{i} \sim \mathcal{C N}\left(\mathbf{0}, \mathbf{A}_{i}-\boldsymbol{\Phi}_{i}\right)$ is the uncorrelated (and also statistically independent) estimation error [24, 22].

\subsubsection{Upstream Transmissions (IoT Networks to SD-RANs)}

Following the above multi-antenna mmWave transmission characterization over a link $i$, the received base-band signal vector $\mathbf{y} \in \mathbb{C}^{M, 1}$ at the BBS at a given instant reads $\mathbf{y}^{(\mathrm{ul})}=\sqrt{P^{(\mathrm{ul})}} \mathbf{H} \mathbf{x}^{(\mathrm{ul})}+\eta^{(\mathrm{ul})}$, where each element of the received signal vector corresponds to a BBS antenna, $\mathbf{H}=\left[\mathbf{h}_{1} \cdots \mathbf{h}_{I}\right] \in \mathbb{C}^{M, I}$, $\mathbf{h}_{i} \in \mathbb{C}^{M, 1}$ denotes the mmWave channel corresponding to the $i$ th SD-GW, $\mathbf{x}^{(\mathrm{ul})}=\left[x_{1} \cdots x_{I}\right]^{\top}$ denotes the $\mathrm{I} \times 1$ vector containing the transmitted signals from all the SD-GWs, $P^{(\mathrm{ul})}$ is the average transmit power of each SD-GW, and $\eta^{(\mathrm{ul})} \sim \mathcal{C N}(0, \sigma)$ is the zero-mean circularly symmetric Gaussian noise with noise power $\sigma^{2}$.

The BBS processes the received signal vector and obtains the estimated channel matrix by multiplying the Hermitian-transpose of the MMSE detection matrix with the signal at the receiver as $\tilde{\mathbf{y}}^{(\mathrm{ul})}=\hat{\mathbf{H}}^{\mathrm{H}} \mathbf{y}^{(\mathrm{ul})}=\hat{\mathbf{H}}^{\mathrm{H}} \mathbf{H} \mathbf{x}^{(\mathrm{ul})}+\hat{\mathbf{H}}^{\mathrm{H}} \eta^{(\mathrm{ul})}$. The $i$ th element of $\tilde{\mathbf{y}}^{(\mathrm{ul})}$ can be written as $\tilde{y}_{i}^{(\mathrm{ul})}=\sqrt{P_{i}^{(\mathrm{ul})}} \hat{\mathbf{h}}_{i}^{\mathrm{H}} \mathbf{H} \mathbf{x}^{(\mathrm{ul})}+\hat{\mathbf{h}}_{i}^{\mathrm{H}} \eta^{(\mathrm{ul})}$, where $\mathbf{h}_{i}$ is the $i$ th column of $\mathbf{H}$. By the elements multiplication, we further get $\tilde{y}_{j}^{(\mathrm{ul})}=\sqrt{P_{i}^{(\mathrm{ul})}} \hat{\mathbf{h}}_{i}^{\mathrm{H}} \mathbf{h}_{i} x_{i}+\sum_{k=1, k \neq i}^{I} \sqrt{P_{k}^{(\mathrm{ul})}} \hat{\mathbf{h}}_{i}^{\mathrm{H}} \mathbf{h}_{i} x_{k}+\hat{\mathbf{h}}_{i}^{\mathrm{H}} \eta^{(\mathrm{ul})}$, where $x_{i}$ denotes the $i$ th element of $\mathbf{x}^{(\mathrm{ul})}$. Then, the signal-to-interference-plus-noise ratio (SINR) achieved by the $i$ th SD-GW, $\gamma_{i}^{(\mathrm{ul})}$, is

$$
\gamma_{i}^{(\mathrm{ul})}=P_{i}^{(\mathrm{ul})}\left|\hat{\mathbf{h}}_{i}^{\mathrm{H}} \mathbf{h}_{i}\right|^{2} /\left(\sum_{k=1, k \neq i}^{I} P_{k}^{(\mathrm{ul})}\left|\hat{\mathbf{h}}_{i}^{\mathrm{H}} \mathbf{h}_{k}\right|^{2}+\left\|\hat{\mathbf{h}}_{i}\right\|^{2} \sigma^{2}\right) .
$$

Assuming an ergodic channel [25, the achievable uplink rate of the $i$ th SDGW is given by

$$
R_{i}^{(\mathrm{ul})}=B \log _{2}\left(1+\gamma_{i}^{(\mathrm{ul})}\right),
$$

where $B$ denotes the wireless transmission bandwidth. We define the uplink sum rate $[\mathrm{bits} / \mathrm{s} / \mathrm{Hz}]$ per cell considering the associations between RRHs and SD-GWs as follows

$$
C^{(\mathrm{ul})}=\sum_{j \in \mathcal{J}} \sum_{i \in \mathcal{I}} g_{i j} N_{i j} R_{i}^{(\mathrm{ul})}
$$



eigenbeamforming, which can be defined as $\mathbf{w}_{i}=\hat{\mathbf{h}}_{i}$ for $i \in \mathcal{I}$.

The associated SINR achieved by the $i$ th SD-GW, $\gamma_{i}^{(\mathrm{dl})}$, is

$$
\gamma_{i}^{(\mathrm{dl})}=v\left|\mathbb{E}\left[\mathbf{h}_{i}^{\mathrm{H}} \hat{\mathbf{h}}_{i}\right]\right|^{2} /\left(\sum_{k=1, k \neq i}^{I} v \mathbb{E}\left[\left|\mathbf{h}_{i}^{\mathrm{H}} \hat{\mathbf{h}}_{k}\right|^{2}\right]+\sigma^{2}\right) .
$$

Since the SD-GWs do not have any channel estimate, we provide an ergodic achievable rate based on the techniques developed in [25, Theorem 1]. Hence, by assuming that the average effective channels $\sqrt{v}\left|\mathbb{E}\left[\mathbf{h}_{i}^{\mathrm{H}} \hat{\mathbf{h}}_{i}\right]\right|^{2}$ can be perfectly learned by the SD-GW, the ergodic achievable spectral efficiency at SD-GW $i$ of $\mathrm{RRH}$ cluster $\mathcal{N}_{i}$ is given as

$$
R_{i}^{(\mathrm{dl})}=B_{i}(1-\kappa) \log _{2}\left(1+\gamma_{i}^{(\mathrm{dl})}\right),
$$

where $B_{i}$ is the bandwidth allocated to the $i$ th SD-GW, $\kappa$ accounts for the spectral efficiency loss due to signaling at RRH.

The downlink sum rate $[\mathrm{bits} / \mathrm{s} / \mathrm{Hz}]$ per cell considering the associations between RRHs and SD-GWs is

$$
C^{(\mathrm{dl})}=\sum_{j \in \mathcal{J}} \sum_{i \in \mathcal{I}} g_{i j} N_{i j} R_{i}^{(\mathrm{dl})} .
$$

\subsection{SD-GWs' QoS Requirements and Optimization Framework}

Besides supporting almost pervasive device connectivity, IoT applications demand services with different rate requirements; therefore, we formulate those 
requirements in terms of SINR coverage and achieved sum-rate per cell at the SD-RAN. We optimize associations between RRHs and SD-GWs so that the SD-GW sum-rate is maximized, and the QoS requirements of SD-GWs and system-level constraints are satisfied simultaneously.

Given $\vartheta$ as the minimum tolerable SINR over a link $i$, the SINR constraints of SD-GWs can be formulated as

$$
\gamma_{i} \geq \vartheta, \forall i \in \mathcal{I}
$$

where $\gamma_{i}$ is computed by either $(9)$ or 12 in case of uplink or downlink transmission, respectively. From the association scheme, we can obtain the equality $q_{j}=1-\prod_{i=1}^{I}\left(1-g_{i j} N_{i j}\right), \forall j \in \mathcal{J}$ and the following sets of association constraints between RRHs and SD-GW:

$$
\begin{gathered}
q_{j} \geq g_{i j} N_{i j}, \forall i \in \mathcal{I}, j \in \mathcal{J} ; \\
\sum_{j=1}^{J} g_{i j} N_{i j} \geq 1, \forall i \in \mathcal{I},
\end{gathered}
$$

where (16) implies that a RRH is in active mode if it is associated with at least one SD-GW whereas 17 ensures that each SD-GW is served by at least one RRH. On the other hand, given the pre-coding vector at the $j$ th $\mathrm{RRH}$ for the $i$ th SD-GW, the transmitter power used by this RRH to serve the $i$ th SD-GW is $\mathbf{w}_{i}^{\mathrm{H}} \mathbf{w}_{i}$ [26]. Let $P_{j}^{(\mathrm{r}-\mathrm{max})}$ denote the maximum power of the $j$ th $\mathrm{RRH}$, we impose the constraints on RRHs' downlink beamforming weights as follows

$$
\begin{gathered}
\sum_{i=1}^{I} \mathbf{w}_{i}^{\mathrm{H}} \mathbf{w}_{i} \leq q_{j} P_{j}^{(\mathrm{r}-\mathrm{max})}, \forall j \in \mathcal{J} ; \\
\mathbf{w}_{i}^{\mathrm{H}} \mathbf{w}_{i} \leq g_{i j} N_{i j} P_{j}^{(\mathrm{r}-\max )}, \forall i \in \mathcal{I}, j \in \mathcal{J},
\end{gathered}
$$

where (18) limits the total transmit power of each RRH and 19 ensures that the transmit power from the $j$ th $\mathrm{RRH}$ to the $i$ th $\mathrm{SD}-\mathrm{GW}$ is set to zero if there is no association between them. Furthermore, by only allowing the links in $\mathcal{N}_{i}$ (see Section 4.2.1) we set the beamforming weights of mmWave communication links as

$$
\mathbf{w}_{i}^{\mathrm{H}} \mathbf{w}_{i}=0 \text { if } N_{i j}=0, \forall i \in \mathcal{I}, j \in \mathcal{J},
$$


so that we reduce all possible links between the $J$ RRHs and the $I$ SD-GWs to $\left|\mathcal{N}_{i}\right|$ links (typically $\left|\mathcal{N}_{i}\right| \ll J I$ ), which in turns dramatically shrinks the size of the possible solution sets of precoding vectors for lower computation complexity [27, 26]. Additionally, the per-fronthaul capacity constraints (neglecting the fronthaul capacity consumption for transferring compressed beamforming vector) are formulated as follows

$$
C \leq C^{(\mathrm{fh})}
$$

where $C$ is computed by 11 in uplink transmission or by 14 in downlink transmission. This indicates that the total data rate transmitted at the each RRH should be less or equal to the rate forwarded by the fronthaul link.

We aim at maximizing the total achievable uplink/downlink data rates at SD-GWs; the overall optimization framework for the SD-RAN becomes

$$
\begin{aligned}
\text { Find } & q_{j} \in\{0,1\}, g_{i j} \in\{0,1\}, P_{i}^{(\mathrm{ul})}, P_{j}^{(\mathrm{dl})}, \mathbf{w}_{i}, \\
& \forall i \in \mathcal{I}, j \in \mathcal{J} \\
\text { maximize } & C=\sum_{i=1}^{I} R_{i}, \\
\text { subject to } & 15,(16,17,18,19,120,12,
\end{aligned}
$$

where $R_{i}$ is calculated based on the communication direction: upstream [see [10]), downstream [see (13)]. The decision variables take values from a discrete set that leads the optimization framework to an integer programming problem. The size of this problem allows the controller to yield a solution in few seconds through exhaustive searching methods. The acquired solutions are then processed by both the BBS pool and the SD-GW for optimal upstream/downstream transmissions.

\section{Numerical Results}

In this section, we present the simulation results of our proposed designs in Section 4.4. In all experiments, each evaluation point represents the average value of $10^{4}$ samples considering the dynamic update of associations in 


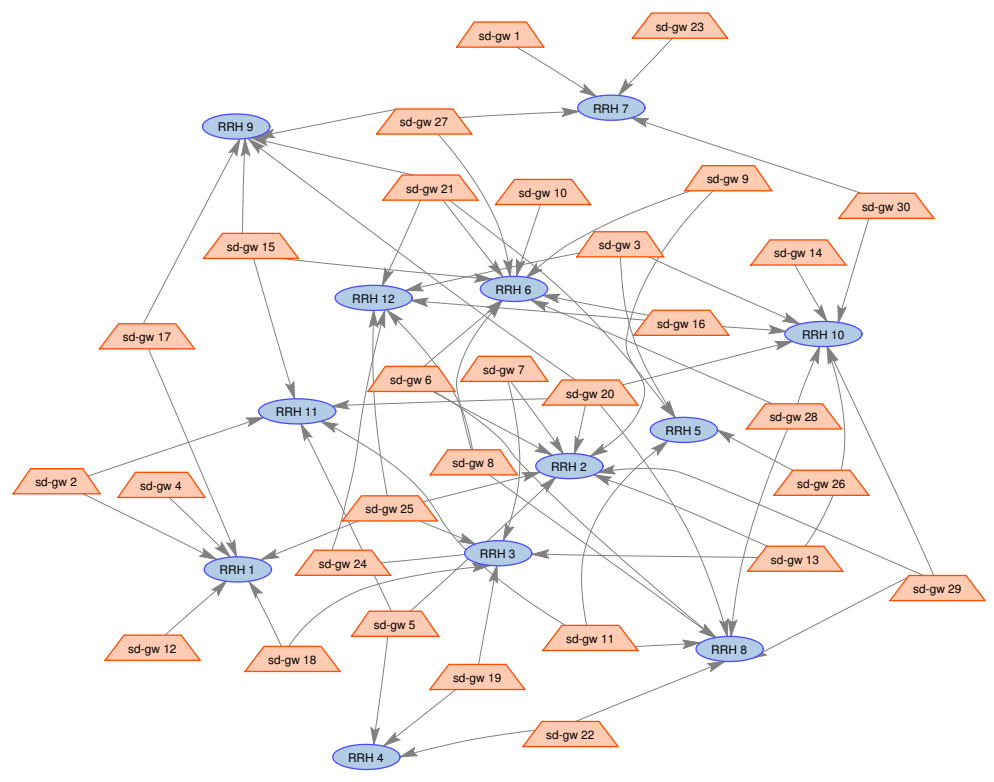

Figure 4: Example of the network topology and association establishment: 12 RRHs that serve $30 \mathrm{SD}-\mathrm{GWs}$ deployed in such an area.

the deployed infrastructure. We examine both the spectral efficiency and the achievable rate per SD-GW in SoftAir. Towards this, we have a set $\mathcal{J}$ of $J=12$ RRHs, each one equipped with $M=4$ antennas; the coverage area of every RRH has a radius of $200[\mathrm{~m}]$. They serve several SD-GW densities ranging from 30 to 100 SD-GWs randomly distributed in the coverage area of RRHs. Fig. 4 illustrates an example of the network topology and the associations established between RRHs and SD-GWs based on the protocol proposed in Section 4.2 .

The wireless bandwidth is set as $B=500[\mathrm{MHz}]$, and the carrier frequency is set as $73[\mathrm{GHz}]$. The channel vectors are generated according to the mmWave communication modeling in Section 4.1, where the three-state path-loss model with log-normal shadowing is considered. The transmit power constraint for each $\mathrm{RRH}$ is $P_{j}^{(\mathrm{r}-\mathrm{max})}=45[\mathrm{dBm}]$. The maximum transmission power of each SD-GW is set as $23[\mathrm{dBm}]$ and the thermal noise power is assumed to be $-101[\mathrm{dBm} / \mathrm{Hz}]$. Moreover, we assume that all RRHs possess the same fronthaul capacity, i.e., $C_{j}^{(\mathrm{fh})}=6[\mathrm{bits} / \mathrm{s} / \mathrm{Hz}], \forall j \in \mathcal{J}$. As 64 QAM is set as the highest 
constellation supported in the system, the maximum spectrum efficiency per improves the achievable downlink rate at SD-GWs whereas the achievable uplink rate remained almost steady. In particular, the network hereby has 12 associated RRHs with 4 antennas; each RRH serves 80 randomly deployed SD-GWs in the coverage area of the RRHs. These results show that there is a trade-off between 


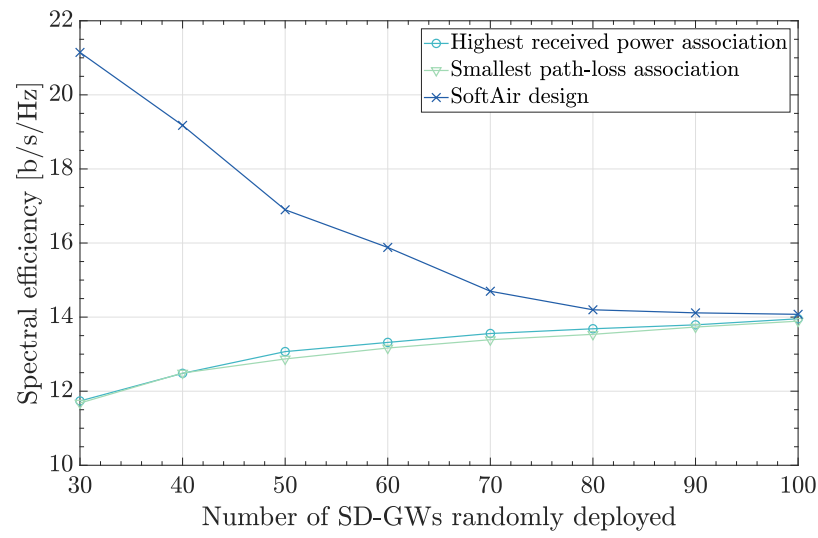

(a)

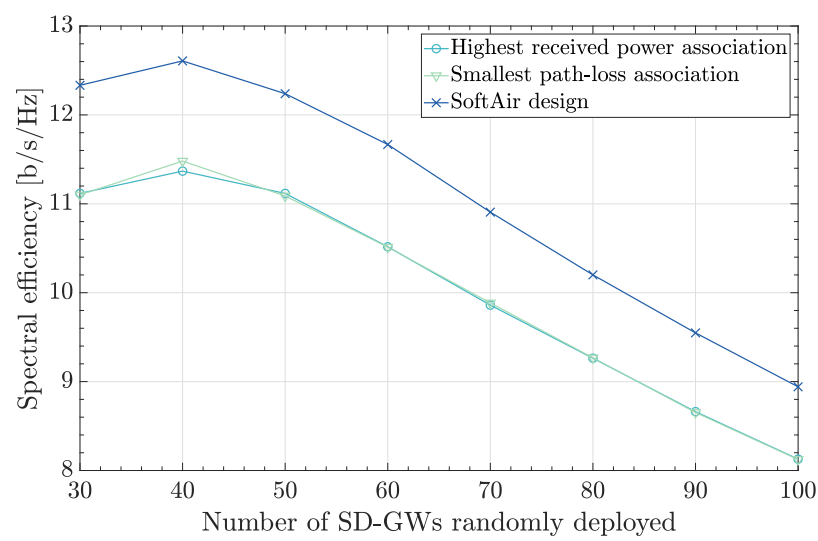

(b)

Figure 5: Spectral Efficiency [b/s/Hz]; 12 RRHs deployed to serve different densities of SDGWs; carrier frequency: $73 \mathrm{GHz}$. (a) Upstream IoT transmissions. (b) Downstream IoT transmissions.

increasing the number of RRHs and increasing the number of antennas at RRHs that affects the achievable rate depending on the direction of the transmission.

To sum up, our SoftAir solution provides ultrahigh data rates (i.e., $430 \mathrm{Mb} / \mathrm{s}$ rate in downlink and $100 \mathrm{Mb} / \mathrm{s}$ rate in uplink through mmWave transmissions) for each SD-GW in densely deployed scenarios, and a decision for increasing the number of RRHs or the number of antennas at RRHs can be made according to the IoT applications. 


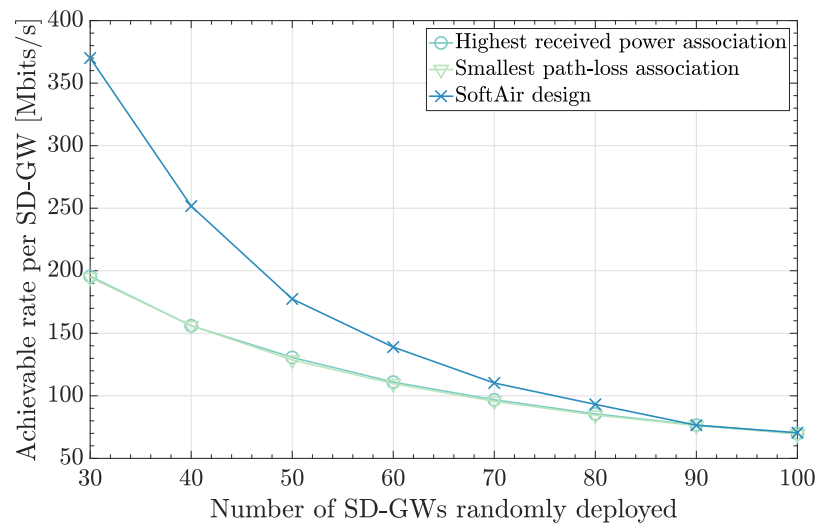

(a)

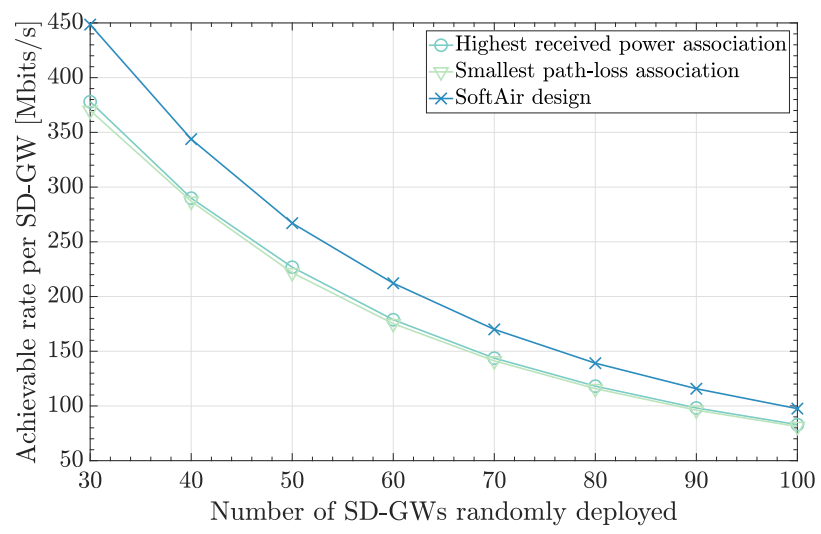

(b)

Figure 6: Achievable rate for the SoftAir design and two existing IoT solutions with conventional mmWave schemes. (a) Upstream IoT transmissions. (b) Downstream IoT transmissions.

\section{Conclusion}

In this paper, we introduced a 5G SoftAir architecture and provided optimal sum-rates for both upstream and downstream IoT communication. First, by jointly exploiting mmWave frontend, MIMO, and virtualization, the SoftAir system is adopted which gives software-defined infrastructure and enables effective coordination among mmWave RRHs. Then, SD-GWs are designed in SoftAir as local controllers that manage and orchestrate Io $\mathrm{T}$ transmissions between IoT 


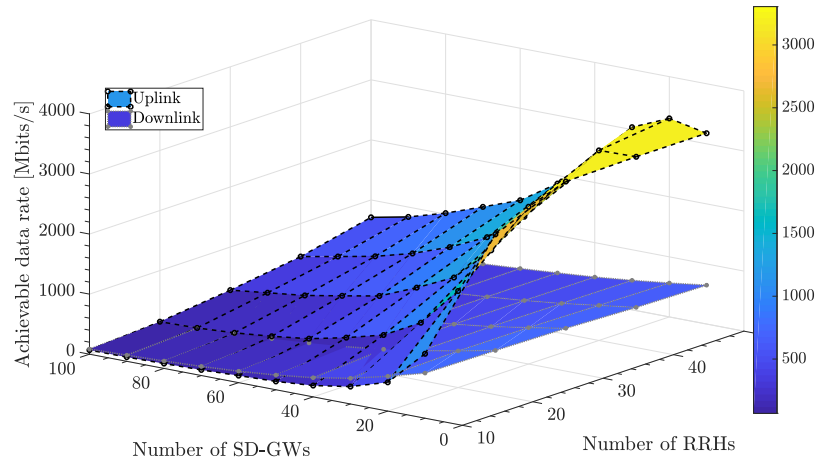

Figure 7: Impact of increasing the number of RRHs on the achievable uplink/downlink rates; the SD-GWs randomly deployed ranges from 10 to 100, and the number of RRHs ranges from 10 to 50 .

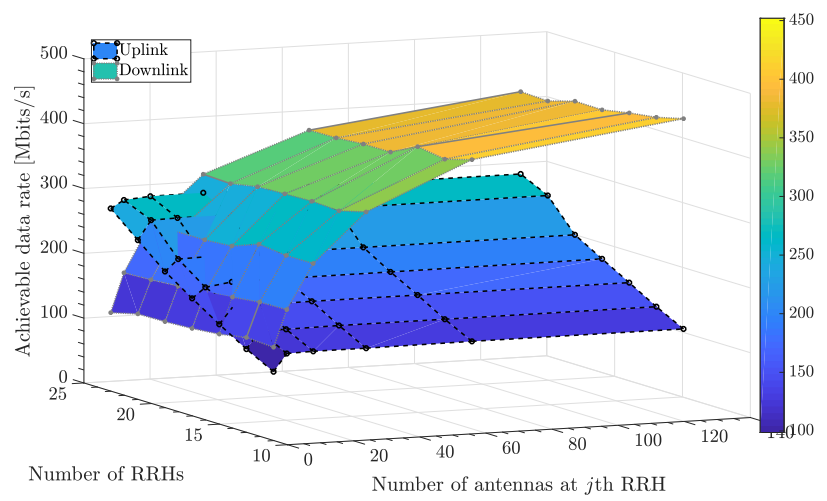

Figure 8: Impact of increasing the number of RRHs or/and antennas at RRHs on the achievable uplink/downlink rates; 80 SD-GWs randomly deployed that are served by a set of RRHs.

networks and SD-RANs. Moreover, a sum-rate optimization framework is proposed in the extended SoftAir, where total data rates of upstream/downstream IoT communication is maximized through optimal associations between mmWave RRHs and SD-GWs. Simulation results validate the superiority of our solutions than conventional IoT schemes, where the extended SoftAir solution achieves optimal spectral efficiency for $5 \mathrm{G}$ IoT communication. 


\section{References}

[1] Ericsson, Ericsson mobility report (Nov. 2017). URL https : //www . ericsson.com/mobility-report

[2] U. Raza, P. Kulkarni, M. Sooriyabandara, Low power wide area networks: An overview, IEEE Commun. Surveys Tuts. PP (99).

[3] I. F. Akyildiz, P. Wang, S.-C. Lin, SoftAir: A software defined networking architecture for 5G wireless systems, Computer Networks 85 (2015) 1 - 18. doi:http://dx.doi.org/10.1016/j.comnet.2015.05.007.

[4] I. F. Akyildiz, S.-C. Lin, P. Wang, Wireless software-defined networks (WSDNs) and network function virtualization (NFV) for $5 \mathrm{G}$ cellular systems: An overview and qualitative evaluation, Computer Networks 93, Part 1 (2015) 66 - 79. doi:http://dx.doi.org/10.1016/j.comnet.2015.10. 013

[5] I. F. Akyildiz, S. Nie, S.-C. Lin, M. Chandrasekaran, 5G roadmap: 10 key enabling technologies, Computer Networks 106 (2016) 17 - 48. doi : http: //dx.doi.org/10.1016/j.comnet.2016.06.010

[6] A. Gran, S. Lin, I. F. Akyildiz, Towards wireless infrastructure-as-a-service (WlaaS) for 5G software-defined cellular systems, in: 2017 IEEE International Conference on Communications (ICC), 2017, pp. 1-6. doi: 10.1109/ICC. 2017.7996597

[7] N. Feamster, J. Rexford, E. Zegura, The road to SDN, Queue 11 (12) (2013) 20.

[8] A. Lara, A. Kolasani, B. Ramamurthy, Network innovation using openflow: A survey, IEEE Commun. Surveys Tuts. 16 (1) (2014) 493-512.

[9] B. A. A. Nunes, M. Mendonca, X.-N. Nguyen, K. Obraczka, T. Turletti, A survey of software-defined networking: Past, present, and future of programmable networks, IEEE Commun. Surveys Tuts. 16 (3) (2014) 16171634. 
[10] A. Al-Fuqaha, M. Guizani, M. Mohammadi, M. Aledhari, M. Ayyash, Internet of things: A survey on enabling technologies, protocols, and applications, IEEE Communications Surveys \& Tutorials 17 (4) (2015) 2347-2376.

[11] M. Noura, M. Atiquzzaman, M. Gaedke, Interoperability in internet of things: Taxonomies and open challenges, Mobile Networks and Applicationsdoi:10.1007/s11036-018-1089-9.

URL https://doi .org/10.1007/s11036-018-1089-9

[12] Z. Qin, G. Denker, C. Giannelli, P. Bellavista, N. Venkatasubramanian, A software defined networking architecture for the Internet-of-Things, in: Network Operations and Management Symposium (NOMS), 2014 IEEE, IEEE, 2014, pp. 1-9.

[13] Y. Jararweh, M. Al-Ayyoub, E. Benkhelifa, M. Vouk, A. Rindos, et al., SDIoT: a software defined based Internet of Things framework, Journal of Ambient Intelligence and Humanized Computing 6 (4) (2015) 453-461.

[14] Y. Yiakoumis, K.-K. Yap, S. Katti, G. Parulkar, N. McKeown, Slicing home networks, in: Proceedings of the 2nd ACM SIGCOMM workshop on Home networks, ACM, 2011, pp. 1-6.

[15] Y. Li, X. Su, J. Riekki, T. Kanter, R. Rahmani, A SDN-based architecture for horizontal Internet of Things services, in: Communications (ICC), 2016 IEEE International Conference on, IEEE, 2016, pp. 1-7.

[16] J. Li, E. Altman, C. Touati, A general SDN-based IoT framework with NVF implementation, ZTE communications 13 (3) (2015) 42-45.

[17] L. Tello-Oquendo, I. F. Akyildiz, S. Lin, V. Pla, SDN-based architecture for providing reliable Internet of Things connectivity in 5G systems, in: 2018 17th Annual Mediterranean Ad Hoc Networking Workshop (Med-Hoc-Net), 2018, pp. 1-8. doi:10.23919/MedHocNet.2018.8407080.

[18] M. R. Akdeniz, Y. Liu, M. K. Samimi, S. Sun, S. Rangan, T. S. Rappaport, E. Erkip, Millimeter wave channel modeling and cellular capac- 
ity evaluation, IEEE J. Sel. Areas Commun. 32 (6) (2014) 1164-1179. doi:10.1109/JSAC.2014.2328154.

[19] M. D. Renzo, Stochastic geometry modeling and analysis of multi-tier millimeter wave cellular networks, IEEE Trans. Wireless Commun. 14 (9) (2015) 5038-5057. doi:10.1109/TWC.2015.2431689.

[20] T. Bai, R. W. Heath, Asymptotic SINR for millimeter wave massive MIMO cellular networks, in: 2015 IEEE 16th International Workshop on Signal Processing Advances in Wireless Communications (SPAWC), 2015, pp. 620-624. doi:10.1109/SPAWC.2015.7227112.

[21] C. Liu, M. Li, I. B. Collings, S. V. Hanly, P. Whiting, et al., Design and analysis of transmit beamforming for millimeter wave base station discovery., IEEE Trans. Wireless Communications 16 (2) (2017) 797-811.

[22] J. Hoydis, S. Ten Brink, M. Debbah, Massive mimo in the $\mathrm{ul} / \mathrm{dl}$ of cellular networks: How many antennas do we need?, IEEE J. Sel. Areas Commun. 31 (2) (2013) 160-171.

[23] S. M. Kay, Fundamentals of statistical signal processing, volume i: Estimation theory (v. 1), PTR Prentice-Hall, Englewood Cliffs.

[24] S.-C. Lin, H. Narasimhan, Towards software-defined massive mimo for 5g\&b spectral-efficient networks, in: 2018 IEEE International Conference on Communications (ICC), IEEE, 2018, pp. 1-6.

[25] J. Jose, A. Ashikhmin, T. L. Marzetta, S. Vishwanath, Pilot contamination and precoding in multi-cell TDD systems, IEEE Trans. Wireless Commun. 10 (8) (2011) 2640-2651. doi:10.1109/TWC.2011.060711.101155

[26] V. N. Ha, L. B. Le, N. D. Dao, Coordinated multipoint transmission design for cloud-rans with limited fronthaul capacity constraints, IEEE Trans. Veh. Technol. 65 (9) (2016) 7432-7447. doi:10.1109/TVT.2015.2485668 
[27] S.-C. Lin, I. F. Akyildiz, Dynamic base station formation for solving NLOS problem in 5G millimeter-wave communication, in: IEEE Conference on Computer Commun. (INFOCOM), 2017, pp. 1-9. doi:10.1109/INFOCOM. 2017.8057227. 